Short Research Communication

\title{
Muscle Extracellular Matrix Scaffold Is a Multipotent Environment
}

\author{
Paola Aulino, 1,2\# , Alessandra Costa1,3,4\# , Ernesto Chiaravalloti ${ }^{2}$, Barbara Perniconi ${ }^{2,5}$, Sergio Adamo ${ }^{1}$, \\ Dario Coletti2, , Massimo Marrelli² , Marco Tatullo2,6\#凶 , Laura Teodori3,7\# \\ 1. Section of Histology and Medical Embryology, Department of Anatomical, Histological, Forensic and Orthopaedic Sciences, Sapienza \\ University of Rome, Rome, Italy \\ 2. Calabrodental clinic, Biomedical Section, Maxillofacial Surgery Unit, Crotone, Italy \\ 3. Fondazione San Raffaele, Ceglie Messapica, Italy \\ 4. Department of Surgery, McGowan Institute, University of Pittsburgh Medical Center, Pittsburgh, PA, USA \\ 5. UMR 8256 CNRS Biology of Adaptation and Aging, University Pierre et Marie Curie Paris 06, Paris, France \\ 6. Tecnologica Research Institute, Biomedical Section, Crotone, Italy \\ 7. UTAPRAD-DIM, ENEA Frascati, Rome, Italy \\ \# These authors equally contributed.
}

$\triangle$ Corresponding author: Dr. Marco Tatullo, PhD, Scientific Director - Tecnologica Research Institute, St. E. Fermi - Crotone, Italy. Phone: 0962-930362; Fax: 0962930362; E-mail: marco.tatullo@tecnologicasrl.com

(C) 2015 Ivyspring International Publisher. Reproduction is permitted for personal, noncommercial use, provided that the article is in whole, unmodified, and properly cited. See http://ivyspring.com/terms for terms and conditions.

Received: 2014.10.08; Accepted: 2014.11.21; Published: 2015.04.06

\begin{abstract}
The multipotency of scaffolds is a new concept. Skeletal muscle acellular scaffolds (MAS) implanted at the interface of Tibialis Anterior/tibial bone and masseter muscle/mandible bone in a murine model were colonized by muscle cells near the host muscle and by bone-cartilaginous tissues near the host bone, thus highlighting the importance of the environment in directing cell homing and differentiation. These results unveil the multipotency of MAS and point to the potential of this new technique as a valuable tool in musculo-skeletal tissue regeneration.
\end{abstract}

Key words: ECM scaffold, tissue engineering, skeletal muscle, bone, cartilage

\section{Brief Communication}

Native extracellular matrix (ECM) scaffold is an emerging tool in tissue engineering for the reconstruction of three-dimensional tissues and organs, respecting their structural and functional features [1]. ECM scaffolds have been demonstrated to recruit stem cells which can contribute to skeletal muscle regeneration, such as the myogenic progenitors CD133+ cells [2], the interstitial stem cells Sca1+/PW1+ [3], and cells presenting general stemness markers such as Sox2, Sca1 and Lin [4, 5]. Recently, it has also been demonstrated that scaffolds from decellularized skeletal muscles promote myogenesis when transplanted in animal models and have the potential to reconstruct skeletal muscle tissue [3]. Whether skeletal muscle acellular scaffolds (MAS) represent a multipotent environment that allows the homing of stem cells and their differentiation towards different cell lineages, depending on their vis a vis microenvironmental signals, is a highly intriguing question. However, the importance of skeletal MAS is underestimated and has received little attention. In order to shed more light on this issue, we decellularized the Tibialis Anterior (TA) as previously described [3] and implanted the derived MAS between the skeletal muscle and the corresponding adjacent bone to analyze the influence of two different, though functionally interconnected, tissues. The TA-derived MAS were implanted: i) at the interface between the TA and tibia bone (TB) and ii) between the masseter (M) and mandible bone (MB) (see Figures 1a and 2a, respectively). This spatial disposition of the implant allowed the simultaneous study of the interaction of 
the scaffold with two different tissues: the skeletal muscle tissue at the interface with the TA or the masseter, where we expected myogenesis [3] and the bone at the interface with the tibia or the mandible, respectively. We chose to use the TA-derived MAS to verify if the capacity to accommodate bone and cartilaginous cells remained intact in both orthotopic sites (tibial muscle on the tibial bone) and in heterotopic sites (tibial muscle on the mandible), for the greater ease both for the collection and both for the graft, of surgical access and for the specific experience in its manipulation in the context of our laboratories. The grafts were analyzed for muscle, cartilage and bone formation 21 days after transplantation. The macroscopic analysis of the TA/TB grafts revealed a white color and a harder consistency (Fig. 1b). Worthy of note was the presence of a hard protrusion on the TB at the contact edge corresponding to the white hard area in the graft; this protrusion is likely to represent bone formation (indicated by circles in Fig. 1c), which was not observed in healthy TB (Fig $1 \mathrm{~d}$ ). At a macroscopic level, the M/MB grafts also displayed a hard white area corresponding to the site of the scaffold implant (indicated by circles in Figs. 2b-d). As expected, the area near the host muscle tissue was colonized by regenerating muscle cells in both the grafts, as demonstrated by the presence of regenerating myofibers (i.e. fibers with a centrally located nucleus) within the scaffold (shown in the insets in Figs. 1e and 2e). Indeed, these MAS constitute a niche that is recognized as being suitable for myogenesis [3]. Strikingly, the areas of the MAS adjacent to the bone were colonized by cartilaginous and bone tissue as well by mononucleated cells in both the grafts. Positive staining for Alizarin red (Figs. 1f and $2 \mathrm{f}$ in TA/TB and $\mathrm{M} / \mathrm{MB}$, respectively) demonstrated the presence of matrix mineralization, which represents the first step in the formation of bone tissue [6, 7], within the MAS in both the grafts in the area near the host bone. Taken together the metachromatic violet staining of toluidine blue (Figs. $1 \mathrm{~g}$ and $2 \mathrm{~g}$ ) and positive staining for Alcian blue (Figs. $1 \mathrm{~h}$ and $2 \mathrm{~h}$ ) showed the presence of cartilaginous matrix within the scaffold in the area near the host bone in both TA/TB and M/MB grafts, respectively. These data demonstrate that MAS represent a suitable environment not only for myogenesis but also for cartilage and bone formation, suggesting that their niche potentials are influenced by their proximity to a specific musculo-skeletal tissue. However, further experiments are needed to clarify whether the scaffold itself is able to promote both myogenesis and bone/cartilage formation or if the scaffold allows the formation of these tissues being influenced by the proximity to the skeletal muscle on one edge and to the bone to the other edge. A recent publication by Perniconi et al. [8] demonstrated that the same scaffold implanted in different anatomical sites, such as the renal capsule and the xiphoid process, didn't promote the formation of any tissue, suggesting that the environment plays a crucial role in the scaffold repopulation.

Our hypothesis is that in our experimental model the scaffold is able to guide migration and differentiation of stem cells which can derive from skeletal muscle, bone, cartilage or circulation, however, the presence of other soluble factors specific of the tissue at the interface with the scaffold (i.e muscle or bone) are necessary to guide stem cell differentiation towards the specific features of that tissue. Furthermore, it has been demonstrated that primary and C2C12 myoblasts can differentiate as bone tissue and that primary and $\mathrm{NIH} / 3 \mathrm{~T} 3$ fibroblasts give rise to cartilage and bone in vitro and in vivo under the influence of specific bone factors (i.e. BMP4) [9], thereby suggesting that different cell populations in skeletal muscle have the potential to develop cartilage and new bone. These findings do not, however, exclude the possibility that this formation of cartilage and bone tissue is due to precursor cells from other anatomical districts, such as the periosteum, which find a suitable environment for differentiation here. Further studies aimed to evaluate the presence and the origin of the cells which contributed to the myogenesis and to the deposition of both mineralized and cartilage matrices will elucidate the mechanism underlying these observations. Clinical applications for bone regeneration are usually based on autologous bone (defined the golden standard), inorganic materials or hydrogels of hyaluronic acid along with other molecular components of the bone ECM, either alone or in combination with mesenchymal stem cells [10], but none has yet proved to be decisive. Although decellularized cartilage ECM is being increasingly used in osteochondral regeneration [11], the value of MAS obtained from different anatomical sites has not yet been considered for bone or cartilage reconstruction.

Our results demonstrate that MAS is, indeed, a promising new technology even in musculo-skeletal regeneration. To the best of our knowledge, only Turner et al. 2011, reported that the small intestine submucosa extracellular matrix (SIS) has the potential to promote cartilage and bone formation in a model of muscle-tendon defect [2]. Sukow et al. previously demonstrated the ability of the same SIS to be osteoconductive in long bone critical defects [12], though neither of these papers explored the possibility of using this approach in musculo-skeletal regeneration. These results highlight the potential of MAS as a multipotent environment that supports the multidirectional differentiation of at least three structurally 
close and functionally interconnected tissues. The importance of these results lies in the fact that the proposed tool may contribute to complex biological processes such as the regeneration of musculo-skeletal tissues. Since our system promotes muscle, bone and cartilage formation at the interface with either long and flat bone, these data pave the way for new clinical approaches to cartilage and bone tissue regeneration in any kind of bone, even if they generate following two different biological process. Flat bone regeneration, in particular, is of considerable interest in oro-maxillo-facial clinical applications. The availability of scaffolds that induce in situ growth and differentiation of bone cells would open the door to exceptional clinical applications in oro-maxillo-facial surgery performed to solve atrophy of the jaws, a widespread condition due to small bone defects or large bone destruction that is a daily clinical problem in oro-maxillo-facial surgery [13]. The gold standard material for this type of tissue regeneration is the autologous bone because it is the only one having osteoinduction, osteoconduction and osteogenesis

Figure 1. Macroscopic and histological evaluation of scaffold transplantation between Tibialis Anterior (TA) muscle and tibia bone 21 days after transplantation. a) Mechanical detachment of TA muscle from tibia bone before the implant. b) The graft 21 days after transplantation. c) Tibia bone after graft removal 21 days after transplantation. d) Untreated tibia bone after TA removal. e) Hematoxylin and eosin staining (H\&E) for whole graft reconstruction shows infiltration by mononucleated cells in the graft and the presence of regenerating myofibers at the edge between the muscle and the graft. The inset represents a micrograph at higher magnification/resolution obtained with a $40 \mathrm{x}$ lens. Scale bar $=50 \mu \mathrm{m}$. f) Alizarin red staining for whole graft reconstruction highlights the presence of calcified areas in the graft. g) and h) Toluidine blue and Alcian staining. respectively, for whole graft reconstruction demonstrate cartilage formation in the graft. Since the images are derived from serial sections, the rectangles indicate corresponding areas in the graft. The insets represent a micrograph at higher magnification/resolution obtained with a $20 x$ lens. Scale bar $=100 \mu \mathrm{m}$.

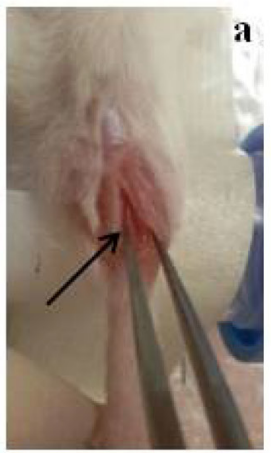

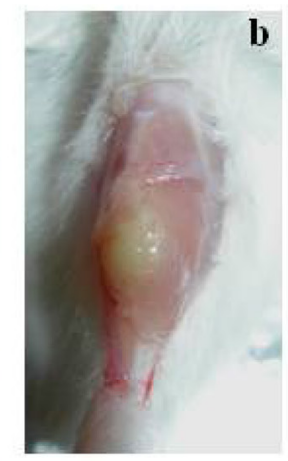
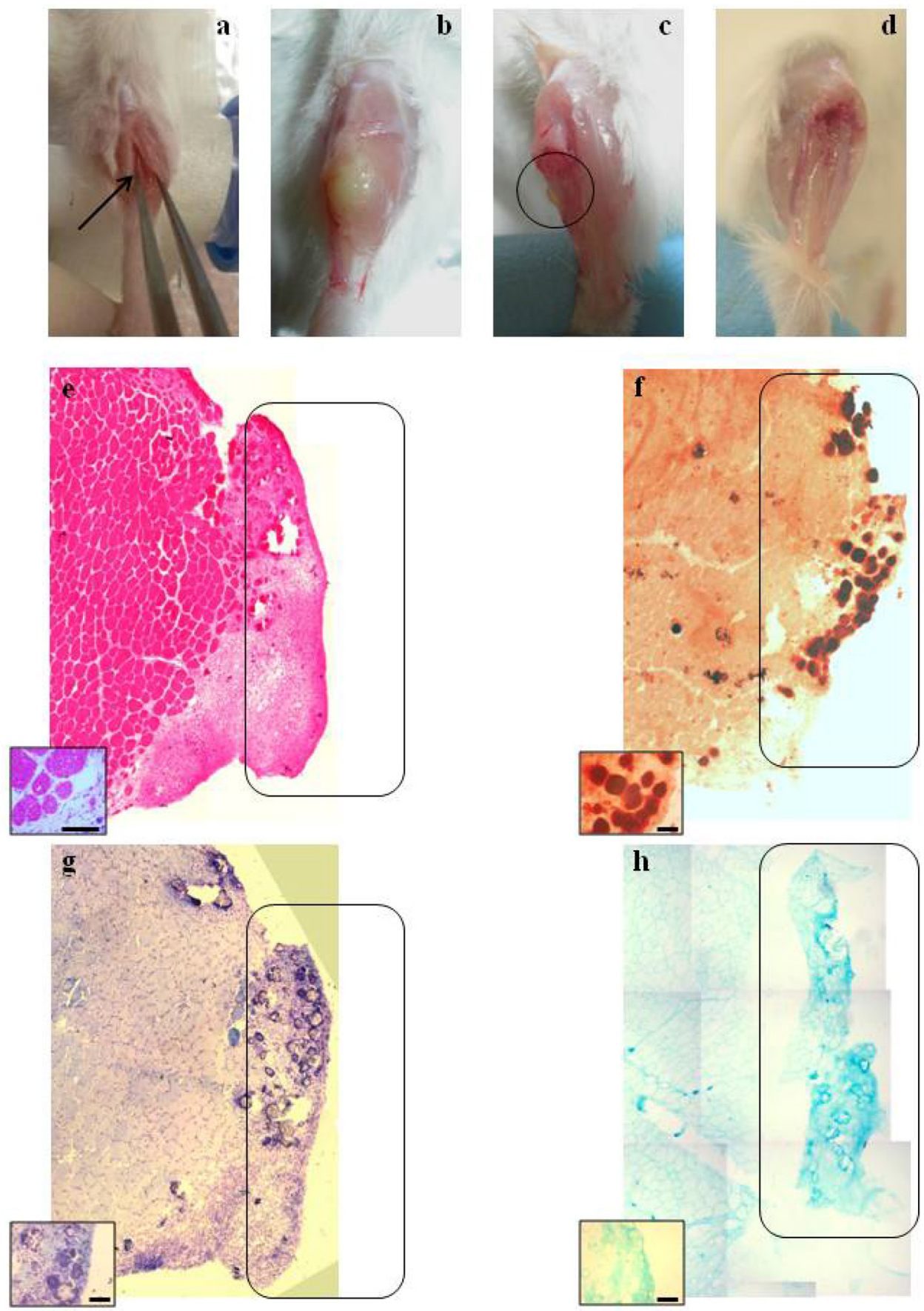

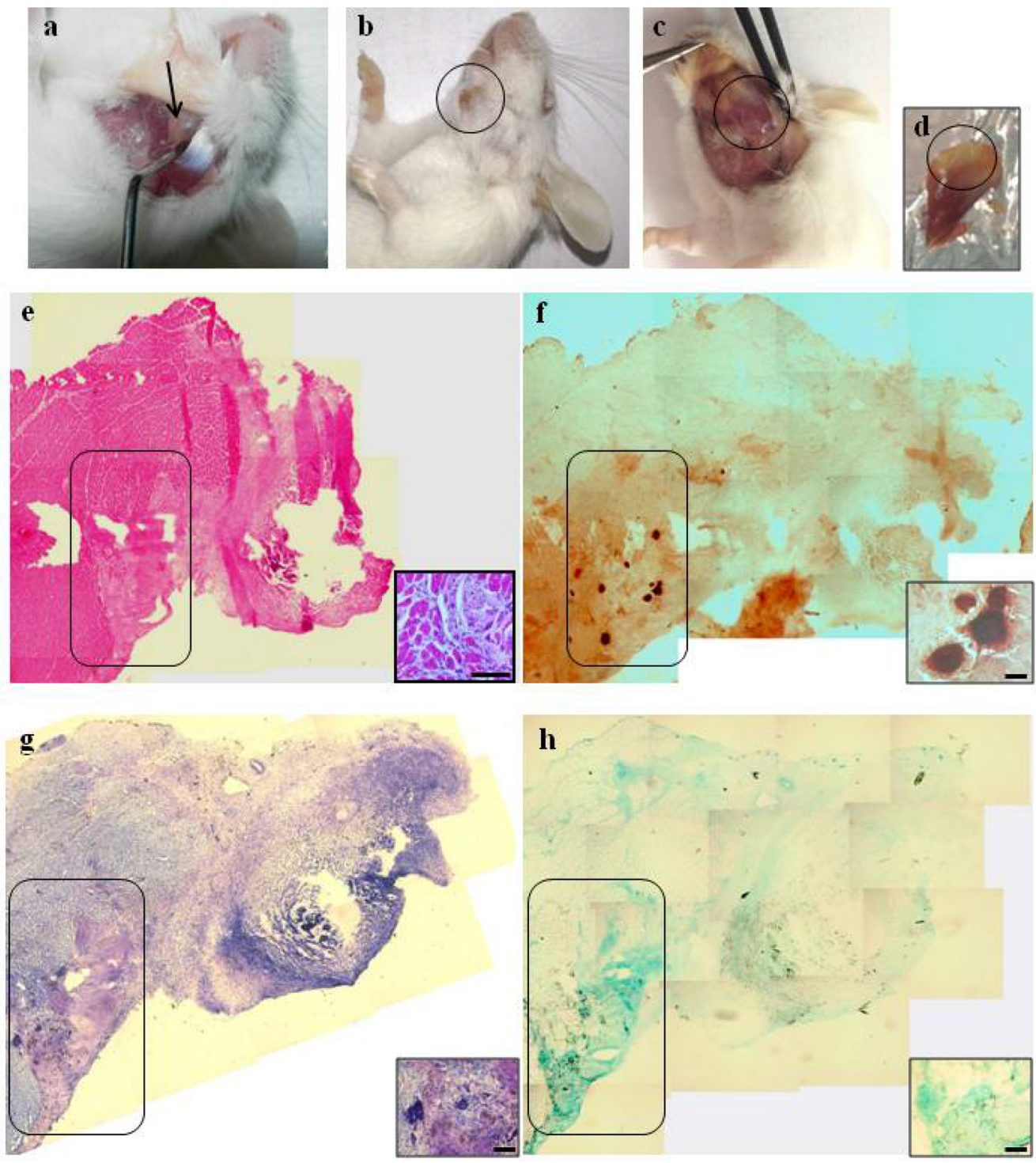

Figure 2. Macroscopic and histological evaluation of scaffold transplantation between masseter (M) and mandible bone (MB) 21 days after transplantation. a) Mechanical detachment of masseter muscle from mandible bone before the implant. b) External surface appearance 21 days after transplantation. c) The graft 21 days after transplantation and d) the collected graft. e) H\&E staining for whole graft reconstruction shows the area of the graft infiltrated by mononucleated cells and the presence of regenerating myofibers at the edge between the muscle and the graft. The inset represents a micrograph at higher magnification/resolution obtained with a $40 \mathrm{x}$ lens. Scale bar $=50 \mu \mathrm{m} f$ ) The same area stained with Alizarin red highlights the presence of calcified areas in the graft. g) and h) Toluidine blue and Alcian staining, respectively, for whole graft reconstruction show cartilage formation in the same area of the graft. Since the images are derived from serial sections, the rectangles indicate corresponding areas in the graft. The insets represent a micrograph at higher magnification/resolution obtained with a $20 x$ lens. Scale bar $=100 \mu \mathrm{m}$.

\section{Materials and Methods}

Experimental overview and surgical procedure Skeletal muscle ECM scaffolds derived from TA muscle of age- and sex-matched inbred mice were implanted in a pocket obtained by detaching i) the TA muscle from the tibia or ii) the masseter (M) muscle from the mandible. Thus in both the sites MAS from TA muscle was used. Following anesthesia with Avertin A (tribromoethanol and 2-methilbutanol from Sigma-Aldrich, St Louis, MO, USA), the skin over the left TA or $M$ was sterilized and the hair removed. An incision was created in the skin layer and the dermal flap opened so as to obtain the pocket described above. Lastly, the skin flap was used to cover the wound and was closed with 3-4 stitches of silk thread (USP 3-0 TT- 26 black silk $45 \mathrm{~cm}$ ). The grafts were collected together with the adjacent muscle 21 days after implantation and subsequently analyzed. In the case of the oro-maxillo-facial, as well, MAS from tibial muscle was used for several reasons, as reported above. Namely: the possibility to verify the accommodating capacity of bone and cartilaginous cells in both orthotropic and heterotopic sites; easier collection and grafting; longer experience in its manipulation in our laboratory.

Animals Three-week-old male BALB/c mice (Charles River) were used in this study. Mice were 
treated according to the guidelines of the Institutional Animal Care and Use Committee. Animals were anesthetized with an intraperitoneal injection of Avertin A (2,2,2-tribromoethanol and 2-methilbutanol from Sigma Aldrich) before implantation. After 21 days, mice were sacrificed by cervical dislocation.

Muscle acellular scaffold (MAS) production ECM-based scaffold was produced as described previously in Perniconi et al. [3]. Briefly, freshly dissected Tibialis Anterior muscles were incubated in sterile 1\% SDS in distilled water for $48 \mathrm{~h}$, at RT under slow rotation. The muscles were then washed in sterile Phosphate Buffered Solution (PBS). Decellularized scaffolds were used for implantation on the same day as they were produced.

Histologic and histochemical analysis Dissected grafts were embedded in Jung tissue freezing medium (Leica, Wetzlar, Germany) and frozen in liquid nitrogen-cooled isopentane. Transverse cryosections of $7 \mu \mathrm{m}$ were obtained using a Leica cryostat. Sections were stained with hematoxylin and eosin (H\&E) using standard methods [15]. Alternatively, cryosections were hydrated for 10 minutes in (PBS) and fixed in 4\% paraformaldehyde for 10 minutes at RT. Sections were then rinsed 3 times in bidistilled water and immersed in Alcian Blue solution (Sigma-Aldrich) pH 2.5 (1 g of Alcian Blue in $100 \mathrm{ml}$ of $3 \%$ acetic acid) for 30 minutes at RT. Lastly, cryosections were rinsed in water, dehydrated in increasing ethanol concentrations and mounted with Eukitt medium. Alternatively, sections were immersed in Alizarin Red solution (Sigma-Aldrich) pH 4.2 (2 g of Alizarin Red in $100 \mathrm{ml}$ of bidistilled water) for 2 minutes at RT. Sections were dehydrated in 1:1 acetone:xylene solution, followed by xylene and finally mounted with Eukitt medium. Serial sections of the same graft were shown in the images. Photomicrographs were obtained using an Axioscop2 plus system equipped with an Axiocam HRc (Zeiss, Oberkochen, Germany) at 1300x1030 pixel resolution.

\section{Acknowledgements}

The research was partially funded by MERIT \#RBNE08HM7T, Fondazione San Raffaele, Ceglie Messapica. Part of the research founds and the post-doctoral fellowships for B.P. and P.A. were provided by Calabrodental in the context of a "PROMETEO Project - Progettazione e Sviluppo di piattaforme tecnologiche innovative ed ottimizzazione di PROcessi per applicazioni in MEdicina rigenerativa in ambito oromaxillofaciale, emaTologico, nEurologico e cardiOlogico" PON01_02834 granted from the Italian MIUR. D. Coletti was supported by ANR (\# 13-BSV1-0005), NIH (\#
5R01CA180057-02), AFM (\# 2012-0773) and by UPMC Emergence 2011.

\section{Competing Interests}

The authors have declared that no competing interest exists.

\section{References}

[1] Faulk DM, Johnson SA, Zhang L, Badylak SF. Role of the extracellular matrix in whole organ engineering. Journal of cellular physiology. 2014;229:984-9.

[2] Turner NJ, Badylak JS, Weber DJ, Badylak SF. Biologic scaffold remodeling in a dog model of complex musculoskeletal injury. The Journal of surgical research. 2012;176:490-502.

[3] Perniconi B, Costa A, Aulino P, Teodori L, Adamo S, Coletti D. The pro-myogenic environment provided by whole organ scale acellular scaffolds from skeletal muscle. Biomaterials. 2011;32:7870-82.

[4] Agrawal V, Kelly J, Tottey S, Daly KA, Johnson SA, Siu BF, et al. An isolated cryptic peptide influences osteogenesis and bone remodeling in an adult mammalian model of digit amputation. Tissue engineering Part A. 2011;17:3033-44.

[5] Agrawal V, Tottey S, Johnson SA, Freund JM, Siu BF, Badylak SF. Recruitment of progenitor cells by an extracellular matrix cryptic peptide in a mouse model of digit amputation. Tissue engineering Part A. 2011;17:2435-43.

[6] Ai-Aql ZS, Alagl AS, Graves DT, Gerstenfeld LC, Einhorn TA. Molecular mechanisms controlling bone formation during fracture healing and distraction osteogenesis. Journal of dental research. 2008;87:107-18.

[7] Varma SR, Sharath Kumar LM, Vidyashankar S, Patki PS. Water Soluble Components of 'Osteocare' Promote Cell Proliferation, Differentiation, and Matrix Mineralization in Human Osteoblast-Like SaOS-2 Cells. Scientia pharmaceutica. 2014;82:375-91.

[8] Perniconi B, Coletti D, Aulino P, Costa A, Aprile P, Santacroce L, et al. Muscle acellular scaffold as a biomaterial: effects on $\mathrm{C} 2 \mathrm{C} 12$ cell differentiation and interaction with the murine host environment. Frontiers in physiology. 2014;5:354.

[9] Li G, Peng H, Corsi K, Usas A, Olshanski A, Huard J. Differential effect of BMP4 on NIH/3T3 and C2C12 cells: implications for endochondral bone formation. Journal of bone and mineral research : the official journal of the American Society for Bone and Mineral Research. 2005;20:1611-23.

[10] Billstrom GH, Blom AW, Larsson S, Beswick AD. Application of scaffolds for bone regeneration strategies: current trends and future directions. Injury. 2013;44 Suppl 1:S28-33.

[11] Benders KE, van Weeren PR, Badylak SF, Saris DB, Dhert WI, Malda J. Extracellular matrix scaffolds for cartilage and bone regeneration. Trends in biotechnology. 2013;31:169-76.

[12] Suckow MA, Voytik-Harbin SL, Terril LA, Badylak SF. Enhanced bone regeneration using porcine small intestinal submucosa. Journal of investigative surgery: the official journal of the Academy of Surgical Research. 1999;12:277-87.

[13] Grimm WD, Dannan A, Giesenhagen B, Schau I, Varga G, Vukovic MA, et al. Translational Research: Palatal-derived Ecto-mesenchymal Stem Cells from Human Palate: A New Hope for Alveolar Bone and Cranio-Facial Bone Reconstruction. International journal of stem cells. 2014;7:23-9.

[14] Barradas AM, Yuan H, van Blitterswijk CA, Habibovic P. Osteoinductive biomaterials: current knowledge of properties, experimental models and biological mechanisms. European cell and Materials, 2011; 21:407-29 .

[15] Fischer AH, Jacobson KA, Rose J, Zeller R. Hematoxylin and eosin staining of tissue and cell sections. CSH protocols. 2008;2008:pdb_prot4986. 